\title{
Reflets
}

Revue ontaroise d'intervention sociale et communautaire

\section{Malgré la rupture, la violence persiste... : La violence conjugale et le harcèlement criminel}

\section{Marise Denault}

Volume 5, numéro 1, printemps 1999

Pratiques et développement économique communautaire

URI : https://id.erudit.org/iderudit/026264ar

DOI : https://doi.org/10.7202/026264ar

Aller au sommaire du numéro

Éditeur(s)

Reflets : Revue ontaroise d'intervention sociale et communautaire

ISSN

1203-4576 (imprimé)

1712-8498 (numérique)

Découvrir la revue

Citer cet article

Denault, M. (1999). Malgré la rupture, la violence persiste... : La violence conjugale et le harcèlement criminel. Reflets, 5(1), 208-225.

https://doi.org/10.7202/026264ar

Tous droits réservés (C) Reflets : Revue ontaroise d'intervention sociale et communautaire, 1999
Ce document est protégé par la loi sur le droit d'auteur. L'utilisation des services d'Érudit (y compris la reproduction) est assujettie à sa politique d'utilisation que vous pouvez consulter en ligne.

https://apropos.erudit.org/fr/usagers/politique-dutilisation/ 


\title{
Malgré la rupture, la violence persiste... La violence conjugale et le harcèlement criminel
}

\author{
Marise Denault \\ Récipiendaire de la bourse RIFAS $1998^{1}$
}

L'élimination totale de la violence conjugale est loin d'être un fait accompli dans notre société. Toutefois, suite à de nombreuses luttes menées par divers groupes de femmes et aux nombreuses recherches effectuées dans les vingt dernières années sur cette problématique, nous savons maintenant qu'il ne s'agit pas d'un problème vécu individuellement par certaines femmes mais bien d'un réel fléau social. En fait, au Canada, de récentes statistiques démontrent que $29 \%$ des femmes, mariées ou l'ayant déjà été, ont été victimes d'agressions perpétrées par leur conjoint (Statistique Canada 1998 :3).

Si nous pouvons maintenant parler ouvertement de violence conjugale, ceci n'a pas toujours été le cas. Un regard historique sur cette question nous permet justement de comprendre le contexte qui a permis à cette violence d'exister et de se perpétuer. Souvenons-nous qu'il n'y a pas si longtemps, lorsqu'une femme se mariait, elle devenait la propriété de son mari. Par ailleurs, en tant qu'épouse, la femme était soumise à l'autorité de son mari qui avait non seulement le droit de la battre mais le devoir de le faire (Mathieu1985:195). Découlant du «devoir de protection» des femmes, les hommes se sont également donné un «devoir de correction» sur celles-ci ainsi que sur leurs enfants (Mathieu1985:195). L'autorité du mari était ainsi inscrite dans les codes religieux, juridiques et sociaux de notre société patriarcale (Dandurand 1994 :4). Par conséquent, tout ce qui se passait entre deux individus mariés était considéré comme étant de nature 
privée et ainsi les règles de l'État qui géraient les individus dans la sphère publique ne s'appliquaient pas (Clark 1989:425).

De son côté,le droit criminel a également collaboré à préserver l'autorité des hommes sur leur épouse. Il existait même une règle nommée «Rule of Thumb» qui encadrait légalement la pratique de la violence conjugale, donnant ainsi aux hommes le droit de battre leur épouse avec un bâton pas plus gros que le diamètre de leur pouce (Côté 1996:91). Ce n'est que vers les années quatre-vingt que l'État reconnut enfin que la violence conjugale constituait un problème nécessitant une intervention et qu'il existe une politique de mise en accusation obligatoire dans les cas de violence conjugale. Toutefois, l'application de cette dernière n'est toujours pas consistante (Rusen 1992:25); une étude démontre même que la moyenne d'arrestations en Ontario n'est que d'une pour chaque deux cas de violence conjugale (Jaffe, Hasting, Reitzel, Austin 1993:90).

Si nous avons désormais des services qui aident les femmes victimes de violence et une certaine reconnaissance de la part du système judiciaire, la sombre réalité reste que même lorsque les femmes violentées quittent leur conjoint, la violence se perpétue suite à la séparation. Ces dernières risquent de se faire harceler, menacer, intimider et même violenter de nouveau par leur exconjoint (Dekeseredy, MacLeod 1997; Johnson, Sacco 1995; Pagelow 1993). La traque, connue sous le terme «stalking», est justement l'une des formes de violence à laquelle sont sujettes les femmes violentées qui quittent leur conjoint (Hall 1997; Pagelow 1997). La traque n'est pas un phénomène nouveau dans notre société mais nous commençons à peine à faire des études sur ce problème en lien avec la violence conjugale. C'est pour cette raison que nous avons décidé de nous pencher sur cette question. Le harcèlement criminel implique plusieurs actions, entre autres, le fait de suivre, de harceler, de menacer, de téléphoner et d'envoyer des choses à son ex-conjointe (Kong 1997; Goode 1995).

C'est particulièrement la problématique du harcèlement criminel et ses liens avec celle de la violence conjugale que nous présenterons dans cet article. Pour ce faire, nous présenterons premièrement les enjeux importants reliés au départ d'une femme 
violentée, une explication du phénomène du harcèlement criminel à partir d'une analyse féministe ainsi que l'implication du système judiciaire dans ce type de cas. Par la suite, nous expliquerons de quelle façon nous avons procédé pour faire notre recherche avant de présenter les résultats de celle-ci et nos conclusions.

\section{La violence conjugale}

La problématique du harcèlement criminel que vivent les femmes après avoir quitté leur conjoint n'est pas une forme de violence qui se situe à l'extérieur de la violence conjugale. Au contraire, il existe des liens très étroits entre ces deux formes de violence. Pour bien comprendre ces liens, il importe d'abord de bien saisir l'épreuve du départ des femmes victimes de violence conjugale.

Selon l'approche féministe, la violence conjugale est un problème d'ordre social qui découle directement de notre société patriarcale. Cette violence n'est qu'une forme individualisée de la domination sociale collective des femmes par les hommes (Regroupement $1994: 18$ ). La violence conjugale se distingue d'autres formes de violence contre les femmes puisqu'elle s'exerce dans le cadre d'une relation privilégiée (Regroupement 1993 :15) et elle s'opère par cycles. Le cycle de la violence est composé de quatre étapes soit, la montée de tension durant laquelle l'homme crée un climat de menace, ensuite vient de violence, qu'elle soit verbale, physique, économique, sexuelle, religieuse ou autre. Puis vient la phase de justification par laquelle l'homme se déresponsabilise face à sa violence et sème un doute chez sa conjointe qu'il tient responsable de cette violence. Enfin, l'homme exprime ses regrets, fait des excuses et promet à sa conjointe de ne plus recommencer. Cette quatrième phase se nomme celle de la rémission ou de la «lune de miel». (Regroupement 1994 :34). Toute relation de couple marquée par la violence comporte des éléments de pouvoir et de contrôle soumettant la femme à son conjoint (Regroupement 1993 :15). Plusieurs femmes tentent de 
mettre fin au cycle de la violence en quittant leur conjoint, se butant ainsi à de nombreux obstacles. Ces obstacles sont non seulement tangibles, tels le manque d'argent, un logement abordable et le fait pour la femme de se retrouver seule avec un ou plusieurs enfants. Ils incluent également la peur de la réaction du conjoint face à la décision de le quitter. Certaines femmes ont même peur d'être tuées par leur conjoint. C'est à cause de cette peur qu'elles n'arrivent pas à quitter, avance Browne (1987:115).

Compte tenu de tous ces enjeux, il n'est pas étonnant que la décision de quitter soit généralement prise suite à un long processus et que celle-ci «...est en perpétuelle évolution» (Cantin, RinfretRaynor 1994 :280). Les femmes qui quittent leur conjoint violent sont en fait plus à risque d'être tuées après une séparation que celles qui restent avec le leur (Kong 1994:4) et ce, plus particulièrement dans les deux mois qui suivent la séparation (Fedorowycz 1997; Wilson, Daly 1993). Une recherche sur les homicides commis entre 1974 et 1992 a démontré que la probabilité qu'une femme soit tuée par son époux était neuf fois plus élevée que celle d'être tuée par un étranger (Wilson, Daly 1994 :4). Évidemment, toutes les femmes qui quittent leur conjoint violent ne sont pas tuées, mais un grand nombre d'entre elles subissent encore de la violence, entre autres, sous forme de harcèlement criminel.

\section{Le harcèlement criminel}

Le harcèlement criminel, la traque, ou le «stalking» est une forme de violence qui consiste en un ensemble de types de harcèlement et de comportements menaçants qui mène une personne à craindre pour sa sécurité (Manitoba Law Reform Commission 1997 :1). La traque n'est donc pas simplement le fait de suivre et de surveiller une personne de façon persistante (Kong 1996 :2), mais elle inclut d'autres formes de harcèlement telles que les menaces, l'intimidation et les téléphones (Goode 1995 :21). Toutes ces actions peuvent paraitre inoffensives mais lorsqu'elles persistent 
contre le gré d'une personne, elles deviennent bouleversantes et dangereuses (Manitoba Law Reform Commission 1997 :1).Ainsi, lorsqu'une femme quitte son conjoint et que ce dernier refuse de mettre fin à la relation, ce qui serait normalement acceptable entre deux personnes consentantes se transforme en une forme de harcèlement criminel (ministère de la Justice 1996). Il existe très peu de données sur l'ampleur du harcèlement criminel. Les statistiques policières du Canada, excluant le Québec, démontrent toutefois que $80 \%$ des victimes de ce crime sont majoritairement des femmes qui sont poursuivies par des hommes (Kong 1997:33). Et, pour $57 \%$ de ces femmes, le harcèlement fut commis par leur ex-conjoint ou ex-mari. Le harcèlement criminel est une forme de violence qui prend le dessus sur la vie entière de la victime puisqu'il détruit son sentiment de sécurité et l'oblige à être continuellement aux aguets. Comme cette forme de violence se produit de façon continue, elle crée chez la victime un sentiment de peur constante (Manitoba Law Reform Commission 1997 :56). Il n'est pas surprenant alors que certaines victimes de ce crime souffrent de dépression, d'abus d'alcool et d'anxiété (Kong 1996:6). Et selon Hall, cette violence peut changer complètement la vie des femmes qui en sont victimes (1997), celles-ci devant, par exemple, déménager et quitter leur emploi, changer leur nom ou disparaître complètement afin d'échapper à leur agresseur.

Voyons maintenant les raisons qui motivent une personne à harceler. D’abord, selon Rosemary Cairns Way (1994), le harcèlement criminel s'inscrit à l'intérieur des nombreuses formes de violence que vivent quotidiennement les femmes au Canada et fait partie des inégalités sociales, économiques et politiques qu'elles subissent. Selon plusieurs, l'élément central qui motive les hommes à traquer leur ex-conjointe est leur désir de contrôle (Bazuwa, Bazuwa 1996; Cairns Way 1994;Hall 1997). De plus, c'est le désir de vengeance et la jalousie qui poussent certains hommes à harceler, selon Hall (1997) et Holmes (1994). Voyons maintenant quels sont les recours judiciaires offerts aux femmes harcelées par leur ex-partenaire. 


\section{Le système judiciaire}

\section{La loi contre le harcèlement criminel}

Depuis 1993, au Canada, une loi inscrite à l'article 264 du Code criminel interdit le harcèlement criminel.La loi C-126 stipule que:

Il est interdit, sauf autorisation légitime, d'agir à l'égard d'une personne sachant qu'elle se sent harcelée ou sans se soucier de ce qu'elle se sente harcelée si l'acte en question a pour effet de lui faire raisonnablement craindre - compte tenu du contexte - pour sa sécurité ou celle d'une de ses connaissances.

Les actes interdits par cette loi sont les suivants:

a) suivre cette personne ou une de ses connaissances de façon répétée;

b) communiquer de façon répétée, même indirectement, avec cette personne ou une de ses connaissances;

c) cerner ou surveiller sa maison d'habitation ou le lieu où cette personne ou une de ces connaissances réside, travaille, exerce son activité professionnelle ou se trouve;

d) se comporter d'une manière menaçante à l'égard de cette personne ou d'un membre de sa famille. (Code criminel 1994 : Art.264.1)

Le harcèlement criminel est une infraction mixte, c'est-à-dire que la Couronne peut choisir d'agir par procédure sommaire ou par acte criminel. Selon la procédure sommaire, la sentence maximale est de six mois d'emprisonnement ou une amende qui ne dépasse pas $2000 \$$ ou encore les deux à la fois. En procédant sous le code criminel, la peine maximale est d'un emprisonnement de cinq ans (Kong 1997: 33).

Au moment de l'adoption de cette loi, plusieurs critiques furent adressées au comité législatif. Entre autres, Rosemary Cairns Way mentionne que divers groupes de femmes ont demandé qu'un 
préambule soit ajouté à cette loi pour contextualiser la réalité des femmes victimes de ce crime (1994:395). Le critère de la «peur raisonnable» fut aussi contesté en ce qu'il entraîne un risque que les femmes se fassent revictimiser par cette loi à cause des stéréotypes sociaux et sexuels (Cairns Way 1994 : 397). De surcroît, la peur de la femme victime pourrait passer pour pathologique (Bazilli 1993 :22-23).Voyons maintenant de quelle façon cette loi est mise en application.

\section{L'application de cette nouvelle loi}

Malheureusement, peu de recherches traitent de la mise en application de la loi canadienne sur le harcèlement criminel. Cependant, les données sur l'application de la politique de mise en accusation et sur le renforcement des ordonnances de garder la paix suggèrent que cette nouvelle loi ne protégera pas nécessairement les femmes violentées (Jaffe, Hastings, Reitzel, Austin 1993; Rigakos 1995). En effet, ces mesures n'empêchent pas nécessairement les hommes d'être violents (Hirschel, Hutchison, Dean 1992; Schmidt, Sherman 1993). Une étude canadienne démontre que dans $19 \%$ des cas, les services de police n'ont pas porté d'accusations même lorsque l'agresseur avait été identifié (Kong $1997: 35$ ).

Les tribunaux jouent également un rôle important dans l'application de cette loi, car les procureurs de la Couronne ont le pouvoir de retirer les inculpations émises par les services de police et de choisir de ne pas poursuivre la personne inculpée. Une étude tenue dans six villes canadiennes démontre que $58 \%$ des inculpations de harcèlement criminel ont été retirées par les procureurs de la Couronne (Gill, Brockman 1996:35). Pour les agresseurs trouvés coupables, $72 \%$ ont reçu une ordonnance de probation et $24 \%$ ont eu une sentence de prison d'une durée de moins de trois mois (Gill, Brockman 1996:42-43). Ces données suggèrent que malgré une loi qui interdit le harcèlement, ce crime n'est pas pris au sérieux par notre appareil judiciaire.

Comme nous pouvons le constater, pour une femme victime de violence, quitter son conjoint violent est une très grande épreuve qui comporte certains risques. Afin de connaitre davantage 
les enjeux du harcèlement criminel nous avons fait une recherche dans le cadre de notre maitrise en travail social. La section suivante portera sur la méthodologie de cette démarche.

\section{Méthodologie de recherche}

Cette recherche qualitative, de type exploratoire, avait pour but premièrement de connaitre davantage les types de violence que vivent les femmes après s'être séparées de leur conjoint. Nous cherchions aussi à savoir pourquoi la violence persiste après la séparation. Finalement, nous voulions connaître l'impact de la nouvelle loi sur le harcèlement criminel, c'est-à-dire les avantages et les désavantages de celle-ci ainsi que la façon dont elle est mise en application. Nous avons préconisé la méthode du groupe focus pour effectuer notre collecte de données. Cette méthode implique le rassemblement d'un groupe d'individus relativement homogène pour discuter d'un sujet donné (Morgan 1988). Notre échantillon comprenait 21 personnes réparties dans quatre groupes, soit une maison d'hébergement, un centre communautaire, un service d'aide aux victimes d'actes criminels et un service de police. Il y avait une moyenne de cinq participantes et participants par groupe et la durée moyenne pour chacune de nos entrevues a été d'une heure trente minutes. Les questions posées aux intervenantes et aux intervenants portaient sur les trois thèmes mentionnés plus haut et, pour ce faire, nous avons élaboré une guide d'entretien. Cette recherche comporte certaines limites, reliées entre autres à la taille de notre échantillon (Mayer, Ouellet 1991:140); ce qui nous empêche de généraliser nos résultats au-delà de la région d'Ottawa-Carleton. De plus, la méthode du groupe focus, qui suscite une interaction et un partage spontané d'informations des membres participant à la discussion (Home 1997:2), peut influencer la direction de la discussion (Krueger 1994 :36). Nous considérons toutefois que cette méthode a permis de recueillir des données pertinentes à nos questions, contribuant ainsi à une meilleure connaissance de ce sujet peu exploré.Voici à présent les résultats de notre étude. 


\section{Les résultats}

\section{Perceptions des intervenantes du harcèlement vécu par les ex-conjointes}

Tout d'abord, nous avons demandé aux intervenantes ${ }^{2}$ de première ligne de s'exprimer sur les types de violence que vivent les femmes après s'être séparées de leur conjoint violent. Nous voulions explorer dans quelle mesure la violence persiste après la rupture, quelle forme elle prend et quelles tactiques sont utilisées par les hommes pour harceler leur ex-conjointe. Les personnes rencontrées nous ont informé que les femmes qui ont quitté leur conjoint vivent plusieurs formes de violence après la rupture, certaines étant de nature très subtile, d'autres beaucoup plus agressives. Selon les intervenantes, les tactiques qu'utilisent les hommes pour harceler leur exconjointe varient d'une situation à l'autre puisque l'homme s'attaque principalement aux "points faibles» de sa conjointe. Un exemple de ceci est le cas de l'homme qui contacte sa conjointe qui l'a récemment quitté, non pas pour la menacer ou l'intimider mais plutôt pour la supplier de revenir ou encore pour lui faire des promesses et lui offrir des cadeaux. C'est qu'il essaie de manipuler son ex-conjointe, explique une intervenante, en faisant ce qu'elle appelle du "chantage émotif». Les écrits sur le harcèlement criminel ne mentionnent pas ces tactiques dites «positives» dont parlent les intervenantes; nous retrouvons tout de même ces dernières dans les écrits sur la violence conjugale. En fait, il semble exister des similitudes entre le harcèlement dit «positif» et la phase de rémission du cycle de la violence conjugale dans laquelle les hommes font des excuses ou achètent des cadeaux afin de se réconcilier avec leur conjointe suite à un incident de violence. On peut se demander alors si cette violence «positive» joue un rôle dans la décision de plusieurs femmes de retourner vivre avec leur conjoint. Nous savons que ces dernières peuvent quitter à maintes reprises un conjoint agressif avant de le faire de façon permanente (Larouche $1987: 59$ ). Selon Ferraro (1997:134), les promesses et les supplications de l'homme 
violent sont un des facteurs qui affectent la décision de la femme violentée de retourner vivre avec celui-ci.

Les intervenantes mentionnent plusieurs autres formes de violence utilisées par les hommes. Entre autres, elles nous informent des menaces de toutes sortes, comme par exemple, brûler la maison, détruire leurs possessions; elles mentionnent que les menaces de mort et de suicide sont aussi très fréquentes.

Nos données suggèrent que les hommes utilisent aussi les enfants pour continuer à avoir un contrôle sur leur ex-conjointe. Ceci se réalise de différentes façons mais la ligne directrice reste la même, c'est que «les enfants sont un sujet de chantage énorme» comme nous dit une intervenante. Selon l'expérience du service de police interviewé, les enfants servent souvent d'excuse pour garder un contact avec la femme et pour continuer à en abuser. À se sujet, Browne $(1997: 68)$ avance que cette tactique est particulièrement efficace puisqu'elle permet aux homme abuseurs de maintenir un contact à long terme avec leur ex-conjointe. Une autre intervenante mentionne qu'il arrive parfois que le père encourage littéralement les enfants à abaisser leur mère, ainsi «c'est elle qui subit encore la violence mais maintenant c'est par l'entremise de ses enfants». La préoccupation des intervenantes que les hommes utilisent les enfants pour essayer de contrôler leur ex-conjointe rejoint les écrits de Jones et Schechter (1992) et de Hart (1990) qui ajoutent que les enfants peuvent aussi être utilisés par l'homme pour se venger de son ex-conjointe.

Les femmes qui quittent leur conjoint n'échappent donc pas à la violence physique et sexuelle, disent les intervenantes qui s'inquiètent aussi du risque que ces femmes se fassent tuer. Une intervenante dit : «souvent c'est pire une femme qui laisse son conjoint violent, et dans certains cas, c'est plus dangereux que de rester avec». Plusieurs études démontrent justement que la violence peut s'intensifier au moment de la séparation (Cantin, RinfretRaynor 1994; Rodgers 1994), augmentant ainsi les risques que les femmes vivent de la violence physique (Johnson, Sacco $1995: 296$ ).

Maintenant que nous connaissons la façon dont les hommes s'y prennent pour harceler leur conjointe, nous présentons les 
données qui démontrent de quelle façon les intervenantes expliquent le harcèlement criminel.

\section{Comment expliquer le harcèlement des ex-conjoints?}

Le lien entre le couple est la première interprétation offerte par les intervenantes pour expliquer le harcèlement criminel. Par cela, les intervenantes sous-entendent, entre autres, tous les liens affectifs qui existent entre la femme et son conjoint, ce qui fait que plusieurs de ces femmes sont encore capables de reconnaître les bons côtés de leur conjoint; ceci les rend notamment plus vulnérables à son contrôle. Les intervenantes constatent aussi qu'il est souvent difficile pour les femmes de mettre un terme à tout l'espoir qu'elles avaient mis dans leur relation. Ceci rejoint Larouche (1987:59) qui explique que la rupture «demeure, dans bien des cas, un geste d'espoir, un moyen pour inciter l'agresseur à changer ses comportements».

Les intervenantes constatent aussi qu'il est difficile pour une femme de mettre un terme à tout l'espoir qu'elle avait mise dans sa relation et l'une d'entre elles affirme que «même si la femme sait très bien qu'elle a pris la bonne décision, son processus de deuil est énorme». Hoff (1990) démontre que les femmes qui quittent leur conjoint doivent justement entreprendre un processus de deuil de leur relation pour se débarrasser de leur souffrance et de leur culpabilité. Par ailleurs, toujours selon Hoff (1990), il est normal qu'une femme soit vulnérable face à son conjoint au moment de la séparation.

Finalement, le manque de ressources financières empêche les femmes de couper complètement les liens avec leur ex-partenaire, augmentant ainsi leur vulnérabilité comme nous l'ont confirmé les intervenantes. Cette réalité touche un grand nombre de femmes au Canada. Selon le Conseil national du bien-être social (1990), une très grande proportion se retrouve dans la pauvreté suite à une séparation ou un divorce.

La persistance de l'ex-conjoint est une autre raison mentionnée par les intervenantes pour expliquer le harcèlement criminel dans ce type de situation. Selon les policiers et policières interviewés, 
«...dans la majorité des cas de harcèlement, l'intention est de se réconcilier». Pour d'autres intervenantes, c'est que les hommes n'acceptent pas que leur conjointe quitte et ils continuent alors d'être violents pour l'en empêcher. Une intervenante résume: "C'est comme si tu m'appartiens, t'es mon objet et tu ne pourras jamais te défaire de moi.J'ai un cadenas à double clé».Ainsi, comme dit une autre intervenante, le harcèlement devient un moyen pour l'homme de garder un certain contrôle sur son ex-conjointe; elle l'explique ainsi «...je sais où tu es, avec qui tu es... et tu ne t'échapperas jamais de moi». Les recherches sur les hommes abuseurs démontrent également que la violence s'explique par un besoin de pouvoir et de contrôle (Currie 1988; Welzer-Lang 1992) tout comme le font certaines recherches sur le harcèlement criminel par l'ex-conjoint (Bernstein 1993, Cairns Way 1994; Hall 1997). Malheureusement, nous trouvons peu de références sur les enjeux de pouvoir et de contrôle des ex-partenaires dans les écrits sur le harcèlement criminel qui, selon Gill et Brockman (1996:42), mettent l'accent plutôt sur les profils psychologiques des hommes qui harcèlent.

Finalement, une autre intervenante constate des situations où les hommes ne veulent pas toujours rétablir leur relation, mais ils harcèlent par vengeance tout simplement, notion mentionnée dans certaines recherches, dont celles de Hall (1997) et de Mahoney (1996).

Voyons maintenant l'impact sur les femmes violentées de la loi canadienne sur le harcèlement criminel.

\section{Impact de la loi}

Notons au départ que les intervenantes de la maison d'hébergement et du centre communautaire interviewées ne connaissaient pas de femmes qui ont eu recours à cette nouvelle loi. Ces intervenantes offrent tout de même leur perception de cette loi, à partir de leur expérience auprès des femmes violentées et du système judiciaire. En revanche, l'application de cette loi était familière aux intervenantes du service d'aide aux victimes et du service de police, compte tenu de la nature de leur travail. 


\section{Aspects positifs de la loi}

Même si peu d'aspects positifs de cette loi furent nommés, il est tout de même important de les mentionner. Une intervenante dit que cette loi a permis d'identifier le phénomène du harcèlement criminel et "cela est un bon début». D'autres intervenantes sont du même avis et ajoutent qu'il était grand temps que notre société reconnaisse cette forme de violence. Un autre avantage est qu'elle permet aux femmes de dénoncer la violence de leur ex-conjoint. Selon une intervenante, «...par le fait de dénoncer la violence de son ex-conjoint, la femme lui montre qu'elle ne lui permet pas d'avoir le pouvoir et le contrôle sur elle». Finalement, selon le service de police interviewé, il est maintenant plus facile de faire des arrestations qu'auparavant; la loi facilite donc leur travail.

Plusieurs aspects nécessitent d'être corrigés pour rendre cette dernière plus efficace.

\section{Aspects à corriger de la loi}

Parmi les inquiétudes des intervenantes face à cette nouvelle loi nous retrouvons des préoccupations reliées à la façon dont la loi est écrite, dont elle est mise en application et le fait que cette loi est peu connue.

Le critère de la crainte raisonnable stipulé dans la loi sur le harcèlement criminel inquiète les intervenantes de la maison d'hébergement et du centre communautaire puisque, selon elles, il porte trop à interprétation. Les femmes qui ont vécu de la violence conjugale et qui par la suite quittent leur conjoint vivent souvent dans la peur. Ainsi, elles se demandent comment les services de police vont s'y prendre pour interpréter cette peur, compte tenu du fait que leur expérience leur a montré que les policiers ont tendance à minimiser la violence vécue par les femmes. Cette même préoccupation a été soulevée par Cairns 
Way $(1994$ :399) qui avance que le critère de la peur raisonnable va justement permettre aux policiers de juger la peur des femmes à partir de stéréotypes sociaux et sexuels.

Le fait que la loi sur le harcèlement criminel soit peu connue inquiète également les intervenantes interviewées en ce qu'il constitue un grand désavantage pour les femmes harcelées par leur exconjoint. Bien souvent, ni la femme victime de violence, ni les instances qui sont censées la défendre ne connaissent la loi et sa mise en application.

Selon plusieurs intervenantes, les services de police ne prennent pas au sérieux la violence que vivent les femmes. Certaines d'entre elles ont même entendu des policiers justifier les actions d'un homme qui harcelait son ex-conjointe. Les intervenantes d'une maison d'hébergement ajoutent que les policiers ont des préjugés parce que comme nous explique l'une d'elles «... la femme quitte et retourne, quitte et retourne, [ainsi] pour les policiers à chaque fois qu'elle appelle, c'est pas important parce qu'elle retourne toujours». Dans une étude canadienne sur le harcèlement criminel, d'autre intervenantes ont soulevé la même inquiétude et ont affirmé que malgré cette loi, la police intervient quand même de façon lente et inadéquate (Gill, Brockman 1996:63). Dans cette même étude, il a aussi été démontré que les policiers étaient réticents à intervenir dans les cas de harcèlement et de violence conjugale, à moins évidemment que le cas leur apparaissait commun (Gill, Brockman 1996:63). Ceci est inquiétant puisqu'une étude démontre que la crainte des femmes de ne pas être prises au sérieux par les service de police est l'une des raisons qui les empêchent de dénoncer la violence qu'elles vivent (Gartner, Macmillan 1995 :398).

\section{Conclusion}

Nous avons résumé les principaux enjeux de la problématique du harcèlement criminel vécu par les femmes violentées qui ont quitté leur conjoint violent. Nous avons entre autres démontré 
que ces femmes sont à risque de se faire harceler et que, conséquemment, il importe de tenir compte des enjeux de pouvoir et de contrôle afin de comprendre à fond cette problématique. C'est-à-dire qu'il existe de nombreux obstacles qui empêchent les femmes de quitter une relation abusive et que celleci est en fait accentuée lorsque la femme subit en plus des représailles de son ex-partenaire. Nous avons aussi démontré que malgré le fait qu'il existe une nouvelle loi sur le harcèlement criminel, celle-ci est peu connue et les intervenantes interviewées s'inquiètent de la façon dont elle sera mise en application. Certaines ont même fait remarquer que le fardeau de la preuve est souvent remis aux femmes, ce qui demande énormément de temps et d'énergie et peut décourager ces dernières à porter plainte.

Cette étude, bien que limitée par sa nature exploratoire, nous a permis de soulever certaines implications pour l'intervention. Tout d'abord, il semble exister un besoin de faire connaître davantage la loi sur le harcèlement criminel, ce qui pourrait se faire entre autres par l'entremise de campagnes de sensibilisation ou par des messages télévisés. Cette publicité permettrait de transmettre le message que le harcèlement criminel n'est pas acceptable dans les cas de violence conjugale et montrerait aux femmes qui sont harcelées qu'il s'agit d'un acte criminel. Enfin, il serait important d'offrir de la formation sur cette problématique aux divers agentes et agents du système judiciaire chargés de l'application de cette loi.

\section{Bibliographie}

BAZILLI, Suzan (1993). Brief to the Legislative Committee on Bill C-126, Toronto, Metro Action Committee on Public Violence Against Women and Children, 112-146.

BAZUWA, Eve et Carl BAZUWA (1996). «Stalking», Domestic Violence :The Criminal Justice Response, London, Sage Publications, 225-240.

BERNSTEIN, Susan (1993). «Living Under Siege: Do Stalking Laws Protect Domestic Violence Victims?», Cardozo Law Review, vol. 15 no 1-2, 525-567.

BROWNE, Angela (1987). When Battered Women Kill, New York, The Free Press.

BROWNE,Angela (1997). «Until Death Do Us Part?», Albert, CARDARELLI (ed.) Violence Between Partners; Patterns, Causes and Effects, Toronto, Allyn and Bacon, ch. 3.

CAIRNS WAY, Rosemary (1994). «The Criminalization of Stalking: An Exercise in Media Manipulation and Political Opportunism», McGill Law Journal, no 39, 379-400. 
CANADA (Ministère de la justice) (1994). Code criminel de poche, Carswell.

CANTIN, Solange et Maryse RINFRET-RAYNOR (s. la dir. de) (1994). "Quitter le conjoint violent comme stratégie de résolution de problème», Violence Conjugale : Recherches sur la violence faite aux femmes en milieu conjugal, Québec, Gaëtan Morin, 264-282.

CLARK, Lorenne (1989). «Feminist Perspectives on Violence Against Women and Children: Psychological, Social Service, and Criminal Justice Concerns», Canadian Journal of Women and the Law, vol. 3, no 2, 420-431.

COMITÉ CANADIEN SUR LA VIOLENCE FAITE AUX FEMMES (1993). Un nouvel horizon: Éliminer la violence Atteindre l'égalité, (Rapport final), Ottawa, Ministère des Approvisionnement et Services Canada.

CONSEIL NATIONAL DU BIEN-ÊTRE SOCIAL (1990). La femme et la pauvreté, dix ans plus tard, Ottawa, Ministère des approvisionnements et services Canada.

CÔTÉ, Andrée (1996). "Violence conjugale, excuses patriarcales et défense de provocation», Criminologie, vol. 24, no 2, 89-113.

CURRIE, David (1988). The Abusive Husband: An Approach to Intervention, Toronto, Health and Welfare Canada.

DANDURAND, Renée (1994). «Femmes et familles: sous le signe du paradoxe», Recherches féministes, vol. 7, no 1,1-22.

DEKESEREDY, Walter et Linda MACLEOD (1997). Woman Abuse : A Sociological Story, Toronto, Harcourt Brace.

DESLAURIERS, Jean-Pierre (1991). Recherche qualitative : guide pratique, Montréal, McGrawHill.

FEDOROWYCZ, Orest (1997). «Homicide in Canada», Juristat, Centre canadien de la statistique juridique, vol. 17, no 9,1-15.

FERRARO, Kathleen (1997). «Battered Women : Strategies for Survival», Albert, CARDARELLI (ed.) Violence Between Intimate Partners: Patterns, Causes, and Effects, Toronto, Allyn and Bacon.

GILL, Richard et Joan BROCKMAN (1996). A review of section 264 (Criminal Harassment) of the Criminal Code of Canada, Working paper, Ottawa, Department of Justice.

GOODE, Mathew (1995). «Stalking: Crime of the Nineties?», Criminal Law Journal, vol. 19, no 1, 21-31.

HALL, Doris (1997). Outside Looking In: Stalkers and Their Victims, Clairmont, California, Ph,D Thesis submitted to the Faculty of Criminal Justice.

HART, Barbara (1993). «Battered Women and the Criminal Justice System», American Behavioral Scientist, vol. 36, no 5, 624-638.

HIRSCHEL, David et Ira, HUTCHISON et Charles, DEAN (1992). «The Failure of Arrest to Deter Spouse Abuse", Journal of Research in Crime and Delinquency, vol. 29, no 1, 7-33.

HOFF, Lee Ann (1990). Battered Women as Survivors, New York, Routledge.

HOLMES, Ronald (1994). "Stalking in America», Law and Order, vol. 42, no 5, 89-92.

HOME, Alice (1997). «Enhancing Research Usefullness with Adapted Focus Groups», Groupwork, vol. 9, no 2, 48-58.

JAFFE, P, E.HASTINGS, D. REITZEL et G.AUSTIN (1993). «The Impact of Police Laying Charges», Legal Responses to Wife Assault ; Current Trends and Evaluation, Newbury Park, Sage publications, ch. 4 . 
JOHNSON, Holly et Vincent SACCO (1995). «Researching Violence Against Women: Statistics Canada's National Survey", Canadian Journal of Criminology, 281-303.

JONES, Ann (1994). Next Time She'll Be Dead: Battering and How to Stop It, Boston, Beacon Press.

JONES, Ann et Susan, SCHECHTER (1994). Quand l'amour ne va plus; échapper à l'emprise d'un conjoint manipulateur, Montréal, Le jour.

KONG, Rebecca (1997). "Le harcèlement criminel au Canada», Tendances sociales canadiennes, Statistique Canada, no 46, 32-36.

KONG, Rebecca (1996). «Criminal Harrassment», Juristat, Canadian Centre for Justice Statistics, Statistics Canada, vol. 16, no 12,1-13.

KRUEGER, Richard (1994). Focus Groups: A Pratical Guide for Applied Research, London, Sage Publishing.

LAROUCHE, Ginette (1987). Agir contre la violence, Québec, La pleine lune.

MAHONEY, Martha (1991). «Legal Images of Battered Women : Redefining the Issue of Seperation", Michigan Law Review, vol. 90, no 1,1-71.

MANITOBA LAW REFORM COMMISSION (1997). Stalking, Report \#98, Winnipeg, Manitoba Law Foundation.

MATHIEU, Nicole-Claude (1985). "Quand céder n'est pas consentir», L'arraisonnement des femmes, Paris, Éditions de l'école des hautes études, 169-234.

MAYER, R et Francine, OUELLET (1991). Méthodologie de recherche pour les intervenants sociaux, Boucherville, Gaëtan Morin.

MINISTÈRE DE LA JUSTICE DU CANADA (1996). Traquer quelqu'un c'est un crime appelé harcèlement criminel, Pamphlet, Ottawa, Ministère des Approvisionnements et Services Canada.

MORGAN, David (1988). Focus Groups as Qualitative Research, Newbury Park, Sage Publications.

PAGELOW, Mildred (1993). «Justice for Victims of Spouse Abuse in Divorce and Child Custody Cases», Violence and Victims, vol. 8, no 1, 69-83.

REGROUPEMENT PROVINCIAL DES MAISONS D'HÉBERGEMENT ET DE TRANSITION POUR FEMMES VICTIMES DE VIOLENCE CONJUGALE (1994). Un grain de sable dans l'engrenage; pistes de solution pour contrer la violence conjugale, Montréal, Bibliothèque nationale du Québec.

REGROUPEMENT PROVINCIAL DES MAISONS D'HÉBERGEMENT ET DE TRANSITION POUR FEMMES VICTIMES DE VIOLENCE CONJUGALE (1993). La violence conjugale... C'est quoi au juste? C'est un moyen de contrôler sa conjointe, Montréal.

RODGERS, Karen (1994). «Résultat d'une enquête nationale sur l'agression contre la conjointe», Juristat, Bulletin de Service, vol. 14, no 9,1-22.

RUSEN, M. (1992). Silencing Their Screams : The Legal Response to Male Battering of Women, Ottawa, National Association of Women and the Law.

SCHMIDT, Janell, et Lawrence, SHERMAN (1993). «Does Arrest Deter Domestic Violence?», American Behavioral Scientist, vol. 36, no 5, 601-609.

WILSON, Margo et Martin, DALY (1993). «Uxoricide in Canada: Demographic Risk Patterns», Canadian Journal of Criminology, vol. 35, no 3, 263-291. 


\section{Note}

1. Octoyée depuis 1998 à une étudiante de l'Université d'Ottawa, la bourse du Regroupement des intervenants contres les agressions sexuelles (RIFAS) vise à souligner l'excellence d'une mémoire de maîtrise en service social portant sur la violence. À chaque année, Reflets plubliera un article rédigé par la récipiendaire.

2. Étant donné que la majorité des personnes interviewées étaient des femmes, le terme «intervenante» sera utilisé tout au long de cette section. 